\title{
Large cervical fibroid: a rare case report
}

\author{
Sunanda N.* \\ Department of Obstetrics and Gynecology, Mysore Medical College and Research Institute, Mysore, Karnataka, India
}

Received: 27 May 2019

Revised: 27 June 2019

Accepted: 02 July 2019

\section{*Correspondence:}

Dr. Sunanda. N,

E-mail: sunanda_n@ rediffmail.com

Copyright: ( ) the author(s), publisher and licensee Medip Academy. This is an open-access article distributed under the terms of the Creative Commons Attribution Non-Commercial License, which permits unrestricted non-commercial use, distribution, and reproduction in any medium, provided the original work is properly cited.

\begin{abstract}
Although leiomyomas are the most common pelvic tumors presenting in the reproductive age group, cervical fibroids are rare accounting for $2 \%$ of all uterine fibroids. We report a case of 40 year old lady presenting with a firm, nontender mass of 22-24 weeks size pregnant uterus with restricted mobility. Laparotomy showed a large mass arising from the anterior lip of cervix, with a small uterus pushed posteriorly. Enucleation followed by total abdominal hysterectomy was done. Large cervical fibroids are rare, presenting with surgical difficulties. Careful dissection by expert hands is needed in the management of such cases.
\end{abstract}

Keywords: Enucleation, Laparotomy, Large cervical fibroid, Mass effect, Magnetic resonance imaging, Ureteric stenting

\section{INTRODUCTION}

The incidence of leiomyoma in the reproductive period is $20 \%$. Only $2 \%$ of these arise from the cervix. ${ }^{1}$ Cervical fibroids are classified depending on their location as anterior, posterior, lateral and central. They can be further classified as interstitial, subserosal and submucosal polypoid. ${ }^{2}$ Large cervical fibroids generally present with pressure symptoms like retention of urine or constipation or sometimes as abdominal mass mimicking ovarian mass. Due to their close proximity, there is increased risk of bladder and bowel injuries along with risk of intraoperative bleeding. Laparotomy is the most common mode as these are difficult to handle and meticulous surgical dissection is important.

\section{CASE REPORT}

A 40 year old multiparous woman presented with history of pain abdomen and white discharge per vagina for last 3 months. She had no menstrual abnormality, urinary retention, urgency, constipation or mass per vagina. There was no history of contact bleeding. General and systemic examination was normal except for mild pallor. On per abdominal examination, a mass of 22-24 weeks size gravid uterus, firm in consistency, non-tender with restricted mobility was noted. Lower margin could not be reached. Per speculum examination showed a large solid mass arising from the anterior lip of cervix with minimal bleeding through cervical so. Bimanual examination showed a large solid mass filling the pelvic cavity extending up to the umbilicus. Uterus could not be felt separate from the mass.

Investigations showed mild anemia with hemoglobin of $8.2 \mathrm{~g} \%$. Ultrasound report showed bulky uterus with large uterine myoma of $14 * 12 * 13 \mathrm{~cm}$. MRI was done which showed large uterine wall myoma involving anterior wall of lower uterine segment with mass effect displacing the bladder without any invasion (Figure 1). Mild bilateral hydroureteronephrosis was seen. There was no evidence of degeneration, calcification or hemorrhage.

As pouch of douglas could not be opened to enucleate the mass vaginally, laparotomy (Figure 2) was done and a large cervical mass $15 * 13 * 11 \mathrm{cms}$ was enucleated 
(Figure 3). Care was taken to isolate the ureters prior to enucleation followed by total abdominal hysterectomy and bilateral salpingo-oophorectomy.

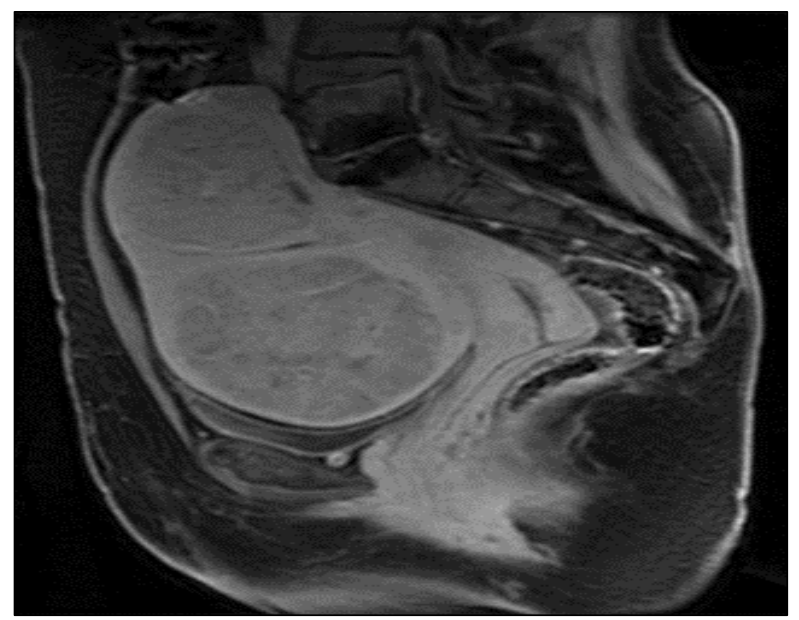

Figure 1: MRI large cervical fibroid.

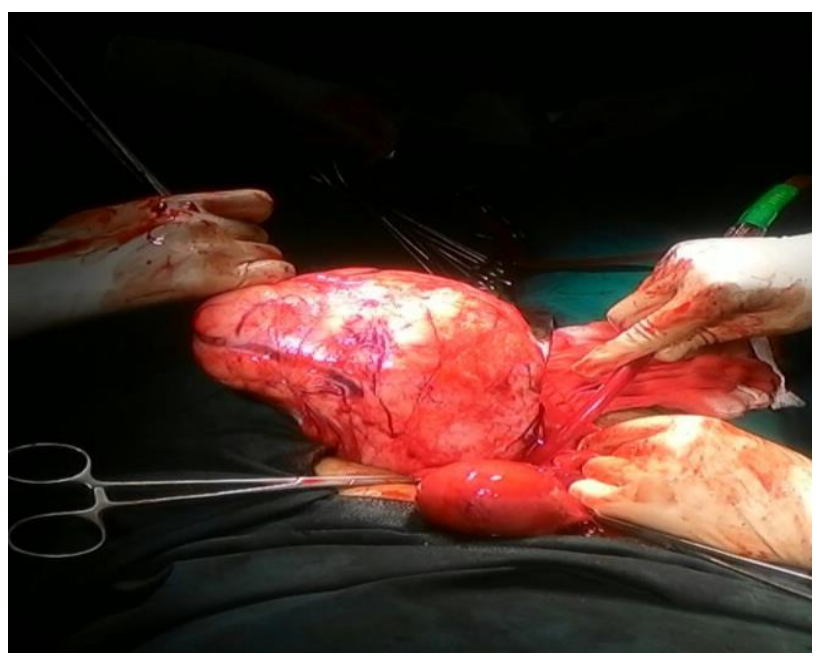

Figure 2: Per-op picture.

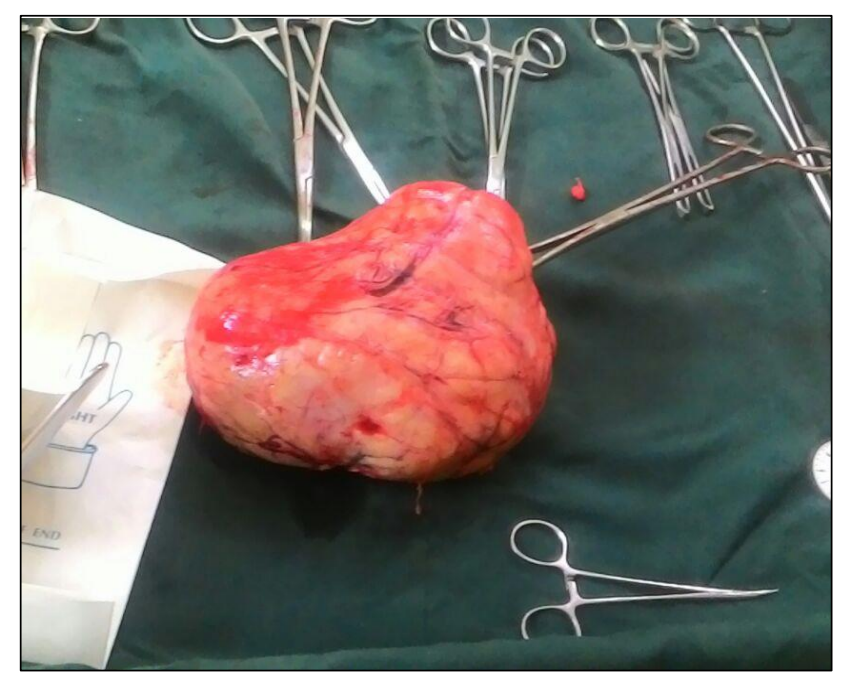

Figure 3: Enucleated myoma.
Post op period was uneventful. Patient was discharged on day 7 after correction of anemia. Histopathology showed leiomyoma (Figure 4) with chronic non-specific cervicitis.

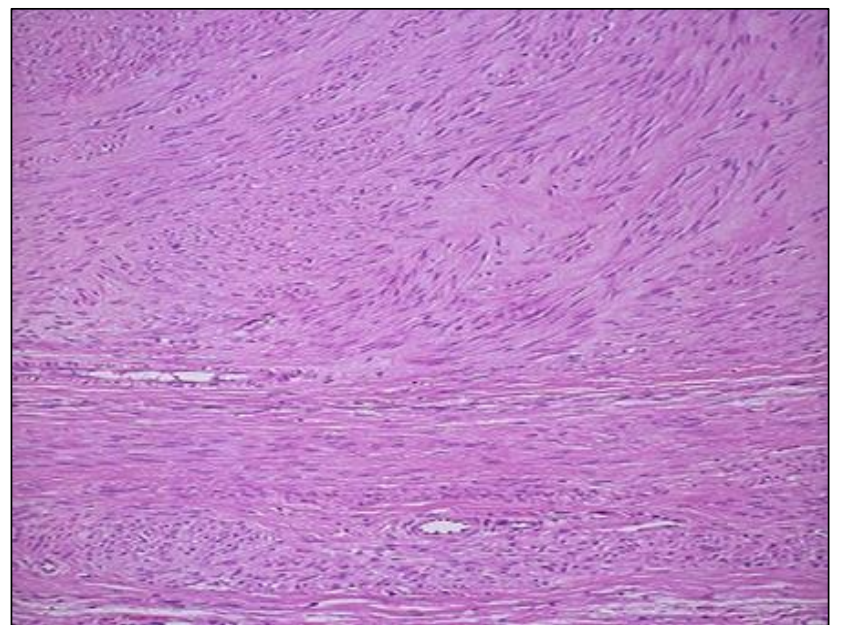

Figure 4: Histopathogy of leiomyoma.

\section{DISCUSSION}

There are very few cases of large cervical fibroids reported in literature. Excessive growths in cervical fibroids are rare. They generally present with symptoms of pressure effects however, in this case the patient was largely asymptomatic except mild abdominal pain.

Unusual presentations of cervical fibroids are also seen. Sushmita sharma and coworkers reported a case of large cervical fibroid of $30 * 26 * 22 \mathrm{cms}$ which presented as abdominal mass. ${ }^{3}$ It was initially confused as an ovarian neoplasm as the fibroid had hyaline and cystic degeneration. Abdominal hysterectomy was the choice of surgery in this case. B Kavitha and coworkers reported a case of large central cervical fibroid with typical "Lantern on top of St. Paul" appearance. ${ }^{4}$ Total abdominal hysterectomy was done along with ureteroureteric anastomosis, bilateral ureteric stenting and bladder repair. ${ }^{3}$

Samal SK et al, reported a case of large cervical fibroid $25 \times 20 \times 15$ with mild hydroureteronephrosis. ${ }^{5}$ Cervical fibroid was enucleated after hemisection. Hemisection of the uterus was done in the relatively avascular mid-line according to Rutherford Morrison's technique with a scalpel and the incision was carried downwards well in to the tumour so that the plane of its capsule is easily distinguished. Ureters were traced and found intact before proceeding with hysterectomy. In this case, patient presented with only vague abdominal pain. Abdominal examination confirmed fibroid although site was not clearly made out. Bimanual examination hinted towards cervical fibroid. Access to pouch of douglas was not possible. In lower uterine fibroids, peritoneal reflection is below the lower border of fibroids unlike cervical 
fibroids where the reflection is above and hence inaccessible. MRI can prove an important diagnostic tool in cases where diagnosis is in doubt.

\section{CONCLUSION}

Treatment of huge cervical fibroids is either by hysterectomy or myomectomy. Myomectomy can be tried in young patients. Pre-operative treatment with GNRH anologues for 3 months can be tried. During surgery, due to proximity of ureters, careful enucleation followed by dissection should be done. During enucleation, limiting dissection to within the capsule is the key to preventing ureteric injury.

Funding: No funding sources Conflict of interest: None declared

Ethical approval: Not required

\section{REFERENCES}

1. Tiltman AJ. Leiomyomas of uterine cervix: A Study of frequency. Int J Gynaecol Pathol. 1998;17(3):2314.
2. Jeffcoate N. Tumors of corpus uteri. In: Bhatla $N$ (ed). Jeffcoate's Principles of Gynaecology, $6^{\text {th }} \mathrm{Ed}$. Delhi, Arnold Publication; 2001:466-497.

3. Sharma S, Pathak N, Goraya SP, Singh A, Mohan P. Large cervical fibroid mimicking an ovarian tumour. Sri lanka J Obstet Gynaecol. 2011;33(1):26-7.

4. Kavitha B, Jyothi R, Rama DA, Madhuri K, Sachin A, Murthy SG. A rare case of central cervical fibroid with characteristic Lantern on top of st. paul appearance. Int J Res Dev Health. 2014;2(1):45-7.

5. Samal SK, Rathod S, Rajsekaran A, Rani R. An unusual presentation of central cervical fibroid: a case report. Int J Res Med Sci. 2014;2(3):1226-8.

Cite this article as: Sunanda N. Large cervical fibroid: a rare case report. Int J Reprod Contracept Obstet Gynecol 2019;8:3414-6. 